\title{
¿Tomar no tomar?: análisis de los motivos de consumo y no consumo de alcohol en jóvenes*
}

\section{To Drink or not to Drink?: Analysis of Alcohol Consumption and non Consumption Reasons in Adolescents}

\author{
Ivonne Andrea Flórez \\ Ángela María Trujillo* \\ Universidad de La Sabana, Colombia \\ Recibido: 20 de febrero de 2012 \\ Revisado: 4 de junio 4 de 2012 \\ Aceptado: 17 de julio 17 de 2012
}

\section{Resumen}

El estudio analiza los motivos de consumo y no consumo en los adolescentes de un colegio de Chía. Se realizó un estudio cualitativo de análisis de contenido en donde se desarrolló una aproximación cualitativa por medio de preguntas abiertas; para posteriormente crear las categorías de análisis y cuantificar los resultados. La aplicación se llevó a cabo en una muestra de 282 adolescentes. Los resultados identifican las categorías y subcategorías de los motivos de consumo, las cuales muestran las diferentes temáticas y cuestiones que los jóvenes describen para argumentar el por qué consumir o no alcohol. La discusión resalta la importancia de generar programas preventivos que tengan en cuenta los motivos de consumo y no consumo de los jóvenes, que tengan en cuenta diferencias individuales en los estudiantes, que promueva los recursos personales como factores de protección y que desestimulen las asociaciones culturales del consumo de alcohol en los jóvenes.

Palabras clave: adolescentes, consumo de alcohol, motivos de consumo, motivos de no consumo, prevención. 


\section{Abstract}

The study analyzes the reasons for consuming alcohol or not among adolescents in a school in Chia. The study has a content analysis qualitative approach, in which open-ended questions were used. Later on, categories of analysis were created and through a quantitative approach results were assessed. The study was conducted on a sample of 282 adolescents. Results identify the categories and subcategories of consuming reasons, which show the various themes and issues that young people described. The discussion highlights the importance of developing prevention programs that take into account the reasons young people have to use or not consume alcohol. These programs should attend to individual differences in students, promote personal resources and protective factors that discourage cultural associations with alcohol use in young people.

Keywords: adolescence, alcohol consumption, reasons for consumption, reasons for non consumption, prevention.

\section{Introducción}

El consumo de alcohol en adolescentes es una preocupación social (Mason et ál., 2011), debido a que abre las puertas al consumo de otras drogas (Trujillo, 2009), aumenta la probabilidad de presentar un consumo problemático de alcohol más adelante (Odgers et ál., 2008), se asocia con conductas delictivas en menores de edad (Sanabria, 2010), con consecuencias negativas como el abuso, la embriaguez, entre otras (Trujillo, Pérez y Scopetta, 2011) y afecta el desarrollo biológico (White, 2003), la salud, (Lara, 2009) y los procesos de aprendizaje y socialización de los jóvenes (Simons-Morton, 2007). Así, se resalta la importancia de realizar estudios que estén dirigidos a aportar información útil en el ámbito de la prevención del consumo de alcohol (Mason et ál., 2011; Odgers et ál., 2008).

Diferentes investigaciones han mostrado que las creencias acerca del consumo del alcohol influyen sobre la decisión de iniciar el uso de esta sustancia (Andrew, Hampson, Barklet, Gerrard y Gibbons, 2008). La teoría del aprendizaje social sostiene que estas creencias se nutren de las experiencias indirectas relacionadas con el consumo, en donde la familia, los amigos, los medios de comunicación y el contexto cultural en el que se desenvuelve el adolescente son las fuentes de las que reciben la información acerca del alcohol (Donovan, Molina y Kelly, 2009; Martino, Collins, Ellickson, Schell y
McCaffrey, 2006). Según las creencias que el adolescente tenga acerca de esta sustancia, se forma una actitud favorable o desfavorable hacia este, y se aumenta o disminuye la probabilidad de que la persona se acerque o no al alcohol (Moral y Ovejero, 2005; Trujillo, Forns y Pérez, 2007).

Diferentes estudios han encontrado que las creencias que los jóvenes tienen del alcohol están distorsionadas. Ya sea positiva o negativamente, la vulnerabilidad frente al alcohol es ilusoria y la percepción se encuentra sesgada por un tinte social y cultural que determina en cierta forma los lugares, los motivos, las personas y, en general, las situaciones en las que los adolescentes consumen o están más dispuestos a consumir (Moral y Ovejero, 2005; Donovan et al, 2009; Litt \&Stock, 2011).

Asimismo se ha identificado que los jóvenes perciben que el alcohol cumple una función de identificación y cohesión grupal, lo cual implica ciertas tradiciones o rituales sociales que justifican su consumo, viéndolo como parte de la vida recreativa y cultural de las personas de su edad (Kloep et ál., 2001; Moral y Ovejero, 2005).

En cuanto a la relación entre la edad de los jóvenes y los motivos de consumo de alcohol, también se han realizado numerosos estudios que muestran que estos se van retroalimentando con la experiencia de consumo, van cambiando conforme 
se genera nueva información y se desarrollan otros procesos de socialización (Andrews et ál., 2008; Gibbons et ál., 2004; Masten, Faden, Zucker y Spear, 2009). En una investigación realizada por Cranford, Zucker, Jester, Puttler y Fitzgerald (2010) se muestra que las expectativas que tienen los jóvenes frente al consumo están en función de la etapa del desarrollo y se convierten en un factor de vulnerabilidad a medida que aumenta la edad. En su estudio se evidencia un cambio de expectativas frente al consumo de alcohol, de negativas a positivas, durante la adolescencia temprana, y se establece que especialmente en los últimos grados del bachillerato, estas expectativas positivas aumentan significativamente.

Asimismo, investigaciones pasadas han mostrado que entre séptimo y octavo grado hay un incremento más acelerado de las expectativas positivas hacia el consumo de alcohol; el grado séptimo representa un punto de transición en la trayectoria general de los motivos y expectativas del consumo de alcohol (Faden, 2006; Oliva, Parra y Sánchez, 2008). Así, los estudios han confirmado que a medida que crecen los adolescentes, hay mayor permisividad social frente al consumo de alcohol, menor percepción de riesgo, mayor percepción de beneficios y mayores oportunidades de consumo. Esto, debido a que al ir creciendo se debilitan las actitudes de oposición al consumo y aumenta la aceptación ante la experimentación con el alcohol (Moral y Ovejero, 2005; Moral, Rodriguez y Sirvent, 2004).

La literatura y las investigaciones encuentran como motivos de consumo en adolescentes la búsqueda de sensaciones, la curiosidad, la vinculación grupal, el escapar de la rutina y el poder experimentar sentimientos de euforia, desinhibición y vigorosidad. Las motivaciones para no consumir se relacionan con la percepción de que es dañino para la salud y de que puede causar accidentes, problemas de dependencia y conflictos económicos o familiares (Comasco et ál., 2010; Bekman et ál., 2011). Se resalta en algunos adolescentes la valoración de la experimentación como fin último, es decir, saciar la curiosidad sin pretender ir más allá de la sola prueba (Moral y Ovejero, 2005).
Aunque se le ha prestado menor atención a los motivos que los adolescentes tienen para no consumir alcohol, estudios recientes muestran que los motivos para no tomar predicen abstinencia, menor uso de alcohol en la adolescencia y una menor prevalencia de consumo en la adultez (Anderson et ál., 2011; Epler, Sher y Piasecki, 2009).

Con base en lo anterior, encontramos que los motivos que los jóvenes tienen para consumir o mantener la abstinencia están relacionados con lo que perciben acerca del alcohol, las expectativas de consumo, las claves contextuales y la percepción de la aprobación de la conducta. Así, esta investigación analiza los motivos de consumo y no consumo en los adolescentes de un colegio de Chía, para construir categorías acerca de las motivaciones que tienen los jóvenes para tomar una decisión de consumo de alcohol a su edad. La investigación se realizó en el mismo colegio, en donde la mayoría de estudiantes comparten variables sociodemográficas familiares y procesos de socialización. Con el fin de observar cómo cambian estos motivos a medida que crecen, encontrar puntos de encuentro en los diferentes cursos y finalmente identificar variables de intervención para la prevención del consumo de alcohol. Este estudio se ubica en la línea de investigación "Salud y Contextos de Interacción" de la Universidad de La Sabana.

\section{Método}

\section{Diseño}

Se utilizó un método cualitativo de análisis de contenido (Pope, Mays, Popay, 2007), donde, a partir de las respuestas dadas por los jóvenes en una serie de preguntas abiertas dirigidas a indagar sobre las motivaciones de consumo y no consumo, se crearon categorías y, finalmente, se cuantificaron los resultados para determinar el número de respuestas de cada curso para cada categoría creada. Se utilizó este tipo de técnica porque permite conocer los aspectos específicos que se querían indagar sin sesgar ni dirigir las respuestas de los estudiantes, identificar las categorías de motivaciones generales que giran alrededor de 
una decisión de consumo del adolescente y comparar las respuestas de los estudiantes de todos los grados.

\section{Participantes}

La muestra de este estudio fue de 282 estudiantes de los grados $5^{\circ}$ de primaria a 11 de bachillerato de un colegio mixto de Chía de estrato 3 y 4 . El rango de edad de la muestra total era de los 9 a los 18 años. La tabla 1 muestra la distribución de los estudiantes y el rango de edad para cada curso. Los participantes fueron contactados por medio del colegio, quien colaboró con el permiso de los padres.

Tabla 1.

Distribución de la muestra según el número de estudiantes y el rango de edad para cada curso

\begin{tabular}{lcc}
\hline Curso & $\begin{array}{c}\text { Número de } \\
\text { estudiantes } \\
\text { entrevistados }\end{array}$ & $\begin{array}{c}\text { Rango de edad de } \\
\text { los estudiantes }\end{array}$ \\
\hline Quinto & 42 & $9-11$ años \\
Sexto & 43 & $11-12$ años \\
Séptimo & 40 & $12-13$ años \\
Octavo & 37 & $13-14$ años \\
Noveno & 43 & $14-15$ años \\
Décimo & 42 & $15-16$ años \\
Once & 42 & $16-18$ años \\
\hline
\end{tabular}

Fuente: autores.

\section{Instrumento}

Se realizó una encuesta de siete preguntas abiertas que permitiera responder a los objetivos del estudio, de las cuales solo se utilizaron las preguntas que representaban las categorías de análisis del estudio: motivos para consumir y motivos para no consumir alcohol. Para el desarrollo de las preguntas se hizo una propuesta inicial por parte de cada una de las investigadoras seguido de una puesta en común. La encuesta muestra validez de contenido, en tanto que las preguntas fueron revisadas por jueces expertos en el tema de consumo de sustancias.
Posteriormente, las pruebas fueron aplicadas a cinco jóvenes de edades similares para determinar si las preguntas se entendían y si permitían obtener respuestas cortas, además de que cumplieran con el objetivo del estudio. No fue necesario modificar ninguna de las preguntas propuestas. El procedimiento de aplicación garantizó que las respuestas de los estudiantes reflejaran cada variable que se quería indagar.

Las preguntas de la encuesta contenían los siguientes enunciados: 1). Piensa en una situación en la que alguien de tu edad (puedes ser tú mismo) se haya negado a consumir alcohol. Describe brevemente cuáles pueden ser las principales razones o motivos por los que esta persona se negó. 2. Piensa en una situación en la que alguien de tu edad (puedes ser tú mismo) haya consumido alcohol. Describe brevemente cuáles pueden ser las principales razones o motivos por los que esta persona lo hizo.

\section{Procedimiento}

Como primera medida, se contactó al colegio en donde se llevó a cabo la investigación. Cumpliendo los lineamientos éticos de la APA, se elaboró un consentimiento informado para que fuera firmado por los padres que accedían a que su hijo(a) participara en el estudio. Una vez se tuvieron los consentimientos informados se inició el proceso de aplicación de la encuesta. El instrumento fue aplicado grupalmente en cada uno de los grados durante una de las horas de clase y fueron realizadas por el mismo entrevistador en todos los casos. Después se transcribieron las respuestas, para primero, identificar los motivos que los estudiantes mencionaban, en donde se tenían en cuenta todos las razones señaladas incluyendo particularidades e idiosincrasias de los estudiantes; segundo; construir las categorías de análisis generales y específicas por cada curso, etiquetando y definiendo cada categoría y subcategorías correspondiente y; tercero, cuantificar los resultados ubicando las respuestas de cada estudiante dentro de las categorías construidas, para finalmente contar las frecuencias de respuestas para cada curso. 


\section{Resultados}

A continuación se presentan los resultados para el análisis de categorías, el sistema de codificación de los motivos de consumo que señalan los jóvenes y las frecuencias de respuestas que se encuentran dentro de cada categoría y subcategoría para cada curso.

\section{Análisis de categorías}

Para la creación de las categorías y subcategorías de los motivos de consumo y no consumo de los adolescentes se clasificaron las respuestas de los estudiantes según los principales motivos mencionados (tablas 1 y 2). Esta categorización de las respuestas dadas por los estudiantes fue llevada a cabo de manera independiente por cada uno de los investigadores, para después discutirlos, determinar las categorías y subcategorías finales $\mathrm{y}$, así, clasificar las respuestas de los estudiantes para determinar las frecuencias.

Como criterios de inclusión para etiquetar, agrupar y mantener o eliminar las categorías y subcategorías se tuvieron en cuenta la frecuencia de los estudiantes por cada subcategoría, la literatura acerca de los motivos de uso y no uso de alcohol en adolescentes y la pertinencia de cada categoría en términos de la relevancia para propuestas preventivas. Es importante tener en cuenta que las categorías y las subcategorías no son excluyentes y que un estudiante podía tener respuestas en más de una subcategoría y categoría. Debido a esto, los porcentajes de las categorías fueron estimados sobre el número total de respuestas y no sobre el número de estudiantes.

\section{Codificación de las motivaciones expresadas por los adolescentes}

Motivos para no consumir: el análisis realizado sobre los diferentes motivos descritos por los que un joven (menor de edad) a su edad no tomaría alcohol dio como resultado ocho categorías. En la tabla 2 se presentan las categorías y las definicio- nes y porcentajes de estudiantes que reportaron respuestas en cada subcategoría.

La categoría recursos personales identifica las respuestas que hacen referencia a motivaciones morales, principios o valores que surgen de la propia ética, creencias religiosas o personalidad del joven y está compuesta por 8 subcategorías (tabla 2). Por otra parte, la categoría normas y leyes hace referencia a todas las descripciones que señalaban como razones para no consumir la presencia de figuras de autoridad, las regulaciones legales y prohibiciones que pueden significar algún tipo de castigo para el joven en el evento que consuma.

La categoría de salud física y mental se define como aquellas razones relacionadas con el daño que el consumo de alcohol pueda generar al organismo y a la salud del estudiante (tabla 2). En la categoría de pérdida de control se agrupan todas las respuestas de los estudiantes que hacen referencia a la decisión de no tomar para evitar problemas personales relacionados con un consumo problemático o el desarrollo de una adicción. La quinta categoría, imagen, incluye todos los motivos relacionados con las consecuencias negativas que el consumo de alcohol puede causar en la imagen social del joven o en lo que puedan pensar los demás de él.

La categoría de miedo contiene las respuestas en las que los estudiantes mencionan esta emoción como razón principal para decidir no consumir. La categoría barreras del momento hacen referencia a circunstancias especiales y particulares de la situación en la que se pueda encontrar el joven y que hace que este se abstenga de consumir alcohol. Por último, en la categoría de no percibe beneficios se agrupan las razones en donde los estudiantes señalan que el alcohol no les despierta ningún interés o que no perciben ninguna ventaja que puedan obtener con su consumo. 
Tabla 2.

Codificación de los motivos por los que un adolescente no tomaría alcohol

\begin{tabular}{|c|c|c|}
\hline \multicolumn{3}{|c|}{ Codificación de los motivos por los que un adolescente no tomaría alcohol relatados por los adolescentes } \\
\hline \multicolumn{3}{|r|}{ Recursos personales } \\
\hline Categoría & $\%$ & Definición \\
\hline Creencias & $10 \%$ & $\begin{array}{l}\text { Se niega a consumir alcohol por su religión, sus costumbres, creencias personales o } \\
\text { por convicción. }\end{array}$ \\
\hline Autoestima & $1 \%$ & Se niega a consumir alcohol porque se quiere y tiene una alta autoestima. \\
\hline Ética & $25 \%$ & $\begin{array}{l}\text { Se niega a consumir alcohol porque considera que es un acto incorrecto, que está } \\
\text { mal, es malo, y que lo correcto es negarse. }\end{array}$ \\
\hline Educación & $11 \%$ & $\begin{array}{l}\text { Se niega a consumir alcohol por la educación que ha recibido, las enseñanzas que } \\
\text { le han inculcado en la casa, o por respeto a la crianza que le han dado sus padres. }\end{array}$ \\
\hline Principios y valores & $16 \%$ & $\begin{array}{l}\text { Se niega a consumir alcohol porque va en contra de sus principios y valores y respeta } \\
\text { su vida y su salud. }\end{array}$ \\
\hline Personalidad & $13 \%$ & $\begin{array}{l}\text { Se niega a consumir alcohol porque es reponsable, autónomo, maduro y tiene el } \\
\text { derecho y la capacidad de decir que no. }\end{array}$ \\
\hline $\begin{array}{l}\text { No quiere seguir } \\
\text { ejemplo familia }\end{array}$ & $1 \%$ & $\begin{array}{l}\text { Cuando el jóven se niega a consumir alcohol porque no quiere seguir el ejemplo de } \\
\text { personas allegadas y familiares. Porque quiere ser diferente. }\end{array}$ \\
\hline Reflexión & $23 \%$ & $\begin{array}{l}\text { Se niega a consumir alcohol porque tiene criterio, es consiente de los daños que } \\
\text { puede causar o reflexiona sobre las consecuencias. }\end{array}$ \\
\hline \multicolumn{3}{|r|}{ Normas y leyes } \\
\hline $\begin{array}{l}\text { Menor edad/pe- } \\
\text { queño }\end{array}$ & $55 \%$ & Se niega a consumir alcohol porque es menor de edad o esta muy pequeño. \\
\hline Prohibiciones & $41 \%$ & $\begin{array}{l}\text { Se niega a consumir alcohol porque está prohibido, no lo dejan, lo castigan, lo rega- } \\
\text { ñan, o personas de autoridad no se lo permiten. }\end{array}$ \\
\hline Conducción & $4 \%$ & Se niega a consumir alcohol porque tiene que manejar. \\
\hline \multicolumn{3}{|r|}{ Salud física y mental } \\
\hline Perjudicial/dañino & $58 \%$ & $\begin{array}{l}\text { Se niega a consumir alcohol porque esta sustancia es dañina, perjudicial, o mala } \\
\text { para el organismo y la salud en general. }\end{array}$ \\
\hline Borrachera & $10 \%$ & Se niega a consumir alcohol porque no se quiere emborrachar o embriagar. \\
\hline Efectos indeseados & $29 \%$ & $\begin{array}{l}\text { Se niega a consumir alcohol para evitar consecuencias físicas negativas y efectos } \\
\text { indeseados de la bebida tales como la muerte, malestar fisico o alguna enfermedad. }\end{array}$ \\
\hline $\begin{array}{l}\text { Problemas psicoló- } \\
\text { gicos o mentales }\end{array}$ & $3 \%$ & $\begin{array}{l}\text { Cuando el joven se niega a consumir alcohol para evitar el desarrollo o el agravio de } \\
\text { algún problema psicológico o una enfermedad mental. }\end{array}$ \\
\hline
\end{tabular}




\section{Codificación de los motivos por los que un adolescente no tomaría alcohol relatados por los adolescentes}

\section{Pérdida de control}

Evitar la adicción $\quad 54 \% \quad$ Se niega a consumir alcohol para no volverse un vicioso, un vago, un alcohólico, un borracho o no desarrollar algun problema con la bebida.

$\begin{array}{lll}\text { Evitar problemas } \quad 42 \% & \begin{array}{l}\text { Se niega a consumir alcohol para evitar accidentes, imprudencias o consecuencias } \\ \text { indeseadas para él u otras personas. }\end{array}\end{array}$

No saben tomar

4\% Se niega a consumir alcohol porque no sabe tomar o no controla la bebida.

Imagen

No aparentar

Por no hacer ridículo

Por lo que puedan pensar los demás

Inexperiencia

Miedo a consecuencias y efectos

Perder el control

A ser descubierto

Ya había tomado

No tiene plata

No quiere (no

tiene ganas)

Está castigado

En ese momento no es adecuado

Gente que no es de su agrado

No tiene motivos para tomar

20\% Se niega a consumir alcohol por que no quiere aparentar o dárselas de algo que no es.

33\% Se niega a consumir alcohol para no hacer el ridículo.

47\% Se niega a consumir alcohol por lo que puedan pensar los demás de él.

\section{Miedo}

$11 \%$ cómo pueda reaccionar.

Se niega a consumir alcohol porque le tiene miedo a los efectos físicos que este $67 \%$ le pueda generar, o a las consecuencias indeseadas, tales como: atentar contra la seguridad personal, causar accidentes o involucrarse en problemas.

13\% Se niega a consumir alcohol porque le da miedo perder el control de sus actos

9\% Se niega a consumir alcohol por miedo a ser descubierto.

\section{Barreras del momento}

2\% Se niega a consumir alcohol porque ya había tomado en otro momento del día.

$2 \% \quad$ Se niega a consumir alcohol porque no tiene plata.

$35 \%$ Se niega a consumir alcohol porque en ese momento no quiere o no tiene ganas.

11\% Se niega a consumir alcohol porque está castigado.

44\% Se niega a consumir alcohol porque no puede en el momento, está tomando medicamentos, está en el colegio o está manejando.

5\% Se niega a consumir alcohol porque está con gente que no es de su agrado o personas en las que no confía.

2\% Cuando el joven no tiene ningún motivo por el cual tomar en ese momento. 


\begin{abstract}
Codificación de los motivos por los que un adolescente no tomaría alcohol relatados por los adolescentes
No percibe beneficios

\begin{tabular}{lrl} 
No lo necesita & $14 \%$ & $\begin{array}{l}\text { Se niega a consumir alcohol porque piensa que no lo necesita, y que puede estar en } \\
\text { una fiesta y disfrutar sin alcohol. }\end{array}$ \\
No le interesa & $16 \%$ & $\begin{array}{l}\text { Se niega a consumir alcohol porque no lo considera importante o no encuentra nada } \\
\text { interesante en su consumo. }\end{array}$ \\
No está de acuerdo & $6 \%$ & $\begin{array}{l}\text { Se niega a consumir alcohol porque no está de acuerdo con su consumo. } \\
\text { Se niega a consumir alcohol porque ya lo probó y no lo quiere volver a hacer, porque } \\
\text { no le gusta }\end{array}$ \\
\hline
\end{tabular}
\end{abstract}

Fuente: autores.

De todas las subcategorías, el motivo más mencionado para que un joven de la misma edad del adolescente no consuma alcohol, si alguien le ofrece, es la de miedo a consecuencias y efectos, con un $67 \%$ de estudiantes que la reportaron. Otras subcategorías mencionadas por más del $50 \%$ de los jóvenes entrevistados fueron: porque no le gusta, es perjudicial/dañino para la salud, porque es menor de edad o es muy pequeño, y para evitar la adicción. Las subcategorías de motivos para no consumir con los menores porcentajes son: autoestima, no seguir el ejemplo de la familia, ya había tomado, no tiene plata, no tiene motivos para tomar, puede causar problemas psicológicos o mentales y no saben tomar.

Motivos para consumir: en la pregunta sobre los motivos por los que un joven de la edad del estudiante toma la decisión de consumir alcohol, cuando alguien le ofrece, se crearon 9 categorías. La tabla 3 incluye los nombres de las categorías creadas y las subcategorías con las definiciones y los porcentajes de estudiantes que las mencionaron.

En la primera categoría, presión externa, están todas las respuestas de los motivos de los estudiantes que se relacionan con la influencia que ejerce una tercera persona o un grupo de personas para persuadirlo, convencerlo o hacer que tomara alcohol. La categoría de imagen hace referencia a los motivos de consumo relacionados con la decisión de tomar alcohol para evitar que los otros piensen mal de él o para proyectar una imagen que puede tener mayor reconocimiento ante los demás. Por su parte, la categoría permisos del momento incluye todas las respuestas que hacen referencia a condiciones particulares, que pueden llevar a que un joven se dé a sí mismo el permiso de tomar alcohol. La quinta categoría, estrategias de afrontamiento, agrupa las respuestas de los estudiantes que mencionan el consumo de alcohol como una opción, para enfrentar situaciones de malestar emocional o manejar los problemas. La categoría beneficios percibidos se compone de las respuestas en las que los jóvenes mencionan una ventaja que puede traer el consumo de alcohol como razón para consumir.

La categoría recursos personales incluye las respuestas que mencionan la ausencia de habilidades y características personales como razones por las que un joven de la edad de ellos puede aceptar tomar alcohol. En cuanto a la categoría de pérdida de control se encuentra referencia a los motivos para consumir, los cuales están relacionados con el nivel de control que tiene el joven sobre la bebida. En cuanto a la categoría de experimentación se incluyen todas las respuestas en las que los estudiantes refieren como motivos de consumo razones relacionadas con el deseo de vivir situaciones o sensaciones nuevas, y querer conocer los efectos del alcohol. Finalmente, la novena categoría, motivos sociales, agrupa todos los motivos de consumo que señalan algún evento o reunión de un grupo de personas como un momento adecuado para aceptar tomar alcohol. 
Con respecto a los motivos para que un joven de la misma edad tome la decisión de consumir, la subcategoría más mencionada por los estudiantes fue influencia de amigos, con un $87 \%$ de adolescentes que la nombran. Las subcategorías referidas por más de la mitad de la muestra son manejo de problemas, porque quiere, disfrutar la fiesta, por probar, le gusta y adicción. Las subcategorías menos nombradas por los estudiantes fueron engaño, brindis, lleva mucho tiempo sin tomar, desaburrirse, relajación, desinhibición, no le importa su salud, embriaguez, e impulsividad (tabla 3).

Tabla 3.

Codificación de los motivos por los que un adolescente tomaría alcohol

Codificación de los motivos por los que un adolescente tomaría alcohol relatados por los adolescentes

\section{Presión externa}

\begin{tabular}{|c|c|c|}
\hline Categoría & $\%$ & Definición \\
\hline Influencia amigos & $87 \%$ & $\begin{array}{l}\text { Decide tomar porque otras personas (amigos o conocidos) le insisten, lo convencen, } \\
\text { lo persuaden, o porque su grupo de pares toman. }\end{array}$ \\
\hline Engaño & $3 \%$ & Es engañado por otra persona y por eso consume alcohol. \\
\hline Lo obligaron & $11 \%$ & Otras personas lo obligaron a consumir alcohol. \\
\hline & & Imagen \\
\hline Aparentar & $37 \%$ & $\begin{array}{l}\text { Decide tomar para aparentar, dárselas, mostrarse como una persona más grande, por } \\
\text { querer ser adulto o proyectar una imagen diferente a la que es la persona. }\end{array}$ \\
\hline Quedar bien & $25 \%$ & $\begin{array}{l}\text { Decide consumir alcohol para quedar bien ante un grupo de personas o ante su grupo } \\
\text { de pares o por no ser humillado, ridiculizado o rechazado. }\end{array}$ \\
\hline Ser aceptado & $19 \%$ & Decide tomar porque así cree que puede ser aceptado dentro de un grupo. \\
\hline Moda/ser popular & $19 \%$ & $\begin{array}{l}\text { Decide consumir alcohol porque es lo que esta de moda o porque tomando puede } \\
\text { ser popular. }\end{array}$ \\
\hline & & Permisos del momento \\
\hline $\begin{array}{l}\text { Lleva mucho tiem- } \\
\text { po sin tomar }\end{array}$ & $5 \%$ & Decide consumir alcohol porque lleva mucho tiempo sin tomar. \\
\hline Porque quiere & $64 \%$ & $\begin{array}{l}\text { Decide tomar porque quiere, lo desea hacer y no percibe ninguna barrera que le } \\
\text { impida consumir alcohol. }\end{array}$ \\
\hline Hacer locuras & $14 \%$ & Decide tomar porque quiere hacer locuras en ese momento. \\
\hline Brindis & $5 \%$ & Decide consumir alcohol para brindar. \\
\hline $\begin{array}{l}\text { Cambiar el } \\
\text { ambiente }\end{array}$ & $14 \%$ & $\begin{array}{l}\text { Decide consumir alcohol porque quiere cambiar el ambiente o sentirse diferente en } \\
\text { el momento. }\end{array}$ \\
\hline
\end{tabular}




\section{Estrategias de afrontamiento}

Manejo de

problemas

Despecho

Diversión

Desaburrirse

Relajacion

Le gusta

Deshinibición

No se quiere (baja autoestima)

Inconsciente

No le importa su salud

No tiene personalidad

No tiene valores o principios

Adicción

Es costumbre

Lo puede controlar

Embriaguez

Por probar

Ignorancia

Impulsividad

Curiosidad de efectos y sabor

$70 \%$

Decide consumir alcohol para afrontar, olvidar o escapar de situaciones difíciles de la vida, problemas o crisis personales.

30\% Decide consumir alcohol porque está despechado y quiere desahogar una pena amorosa.

\section{Beneficios percibidos}

$33 \%$

$3 \%$

$3 \%$

$61 \%$

$2 \%$
Decide consumir alcohol para divertirse, pasarla mejor, pasarla bien, pasar un buen rato, estar feliz y estar chévere

Dedide consumir alcohol para desaburrirse, por distracción, porque no tiene nada más que hacer o porque está desparchado.

Decide consumir alcohol para relajarse, desestresarse o tranquilizarse.

Decide consumir alcohol por placer porque le gusta la sensación que le produce o porque le gusta el sabor de la bebida.

Decide consumir alcohol para expresarse, tener más seguridad en sí mismo, coger valor o arriesgarse a hacer algo que se le dificulta hacer sin alcohol.

\section{Recursos personales}

Decide consumir alcohol porque no se quiere, no se respeta o tiene baja autoestima.

Aunque conoce las consecuencias negativas del consumo de alcohol, decide tomar.

No le importa su salud y por eso consume alcohol.

Decide consumir porque es una persona que no tiene personalidad, madurez o responsabilidad.

Es una persona sin valores o principios y por eso acepta consumir alcohol.

\section{Pérdida de control}

Decide consumir alcohol porque tiene problemas con el trago, es alcohólico, un vicioso o un borracho, y no puede negarse a tomar.

Está acostumbrado a consumir alcohol y por eso decide aceptarlo.

La persona sabe que puede controlar el consumo y por eso decide tomar.

Decide consumir alcohol porque se quiere emborrachar.

\section{Experimentación}

Decide consumir alcohol porque quiere probarlo.

Decide consumir alcohol porque no conoce cuáles son las consecuencias negativas de su consumo; por ignorante.

Decide consumir alcohol porque es una persona impulsiva y no pensó en lo que estaba haciendo.

Decide consumir alcohol porque le atrajo, porque quiere experimentar nuevas cosas o tiene curiosidad acerca de sus efectos y sabor. 
Disfrutar la fiesta $\quad 63 \% \quad$ Decide consumir alcohol para compartir con sus amigos, disfrutar la fiesta, entrar en ambiente o prender e iniciar la fiesta.

Eventos sociales $\quad 16 \%$ Acepta consumir alcohol porque es un evento social.

Ejemplo social $\quad 22 \% \quad$ Decide consumir alcohol porque todo el mundo lo hace y ese es el ejemplo que dan en la sociedad.

Fuente: autores.

\section{Motivos más frecuentes descritos por los jóvenes}

El análisis de las categorías propuestas en esta investigación permitió determinar qué motivos eran los más nombrados por los jóvenes en general, tanto para tomar como para no tomar alcohol. Así, como puede observarse en la figura 1, se encontró que de las 624 posibles respuestas dadas por los jóvenes en cuanto a las motivaciones para no consumir alcohol, el $27 \%$ hacen referencia a los recursos personales. Por su parte, de las 545 respuestas dadas por los jóvenes con respecto a las motivaciones por las que una persona menor de edad consumiría alcohol, el $22 \%$ hacen referencia a beneficios percibidos, el $20 \%$ hacen referencia a la imagen.
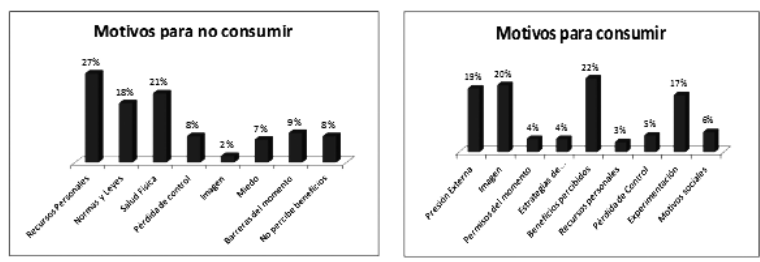

Figura 1. Porcentajes de las categorías de motivos de consumo y no consumo para toda la muestra.

\section{Análisis por curso}

Análisis descriptivo de las categorías según el curso: La tabla 4 muestra los porcentajes, de las respuestas encontradas para no consumir, para las categorías en cada curso. Como puede observarse, en el grado quinto la categoría más mencionada fue la de salud física, en los grados sexto y séptimo la de pérdida de control, en el grado octavo la de miedo, en los grados noveno y décimo la imagen, y en el grado once la de barreras del momento.

En cuanto a las motivaciones para no consumir alcohol se encontró que la categoría de recursos personales disminuye de quinto a octavo y vuelve a aumentar en noveno, con un porcentaje mayor que en los grupos anteriores, siendo octavo un curso de inflexión entre séptimo y noveno. Los estudiantes que más mencionaban los recursos personales tales como la ética, la personalidad, los principios y valores como razones para no consumir son los de once. Los estudiantes que menos nombraron esta categoría fueron los de octavo grado. En cuanto a la categoría de normas y leyes se encuentra que, entre quinto y noveno, los porcentajes se mantienen en un rango del 13 al 19\%, entre noveno y décimo se presenta una disminución del $9 \%$ y entre décimo y once una disminución del 4\%; en los dos últimos cursos se mencionan menos las normas y las leyes como motivos para no consumir. Los cursos que más nombran la categoría de normas y leyes son los de sexto y noveno.

La categoría de salud física tiene los mayores porcentajes de respuesta en los cursos de quinto, sexto y décimo y los menores porcentajes en los cursos de noveno y once. En los primeros cursos se observa una tendencia a que a medida que aumenta el curso disminuye la categoría de salud física como motivo para no consumir; sin embargo, esta tendencia varía en octavo y décimo en donde se presenta un aumento en las respuestas 
relacionadas con el daño a la salud. En la categoría de pérdida de control se observa una disminución significativa entre el paso de noveno a décimo, en donde los cursos de décimo y once presentan el menor porcentaje de respuestas relacionadas con esta categoría. En los cursos de quinto a noveno los porcentajes se mantienen en un rango del $19 \%$ al $23 \%$, de nuevo con la excepción de octavo que se tiende a acercar más a los porcentajes de los cursos mayores. Los resultados muestran que en los cursos de octavo, décimo y once se reconoce menos la posibilidad de que el alcohol pueda generar adicción o problemas como un motivo para no consumir.

Se observa que los cursos de noveno, décimo y once le dan más peso a que la imagen se pueda afectar de manera negativa como motivo para no consumir. En cambio, en los cursos de quinto a octavo esa es una razón que casi no se menciona y que tiene poco peso para una decisión de no consumo. El tema de la imagen aparece hasta el grado séptimo como motivo de no consumo. La categoría de miedo adquiere mayor importancia a partir del grado octavo, llegando a un mayor porcentaje en el grado décimo.

La categoría barreras del momento, al igual que con la categoría de imagen, comienza a ser mencionada a partir del grado séptimo, aumentando entre el grado sexto y séptimo, disminuyendo en un menor porcentaje hasta el grado décimo y llegando al punto más alto en el grado once. Los estudiantes de quinto y sexto no reconocen los impedimentos circunstanciales y temporales como motivos de no consumo. Para los estudiantes de once, en cambio, las razones asociadas con circunstancias excepcionales que puedan impedir el consumo de alcohol son las más mencionadas. Finalmente, la categoría de no percibe beneficios, comienza a cobrar fuerza en el grado noveno, aumentando casi el doble en el grado décimo y disminuyendo de nuevo en el grado once. Esta categoría es poco mencionada por los estudiantes de quinto a octavo, evidenciando una baja asociación entre la abstinencia y la baja percepción de beneficios.
Tabla 4.

Porcentajes de cada curso en las categorías de motivos para no consumir categoría

\begin{tabular}{|c|c|c|c|c|c|c|c|}
\hline \multirow[t]{2}{*}{ Categoría } & \multicolumn{7}{|c|}{ Curso } \\
\hline & 5to & 6to & $7 \mathrm{mo}$ & $8 \mathrm{vo}$ & 9no & $10 \mathrm{mo}$ & 11 \\
\hline $\begin{array}{l}\text { Recursos } \\
\text { personales }\end{array}$ & $14 \%$ & $12 \%$ & $11 \%$ & $6 \%$ & $16 \%$ & $19 \%$ & $22 \%$ \\
\hline $\begin{array}{l}\text { Normas y } \\
\text { leyes }\end{array}$ & $15 \%$ & $19 \%$ & $13 \%$ & $17 \%$ & $19 \%$ & $10 \%$ & $6 \%$ \\
\hline Salud física & $20 \%$ & $18 \%$ & $14 \%$ & $15 \%$ & $9 \%$ & $18 \%$ & $8 \%$ \\
\hline $\begin{array}{l}\text { Pérdida de } \\
\text { control }\end{array}$ & $19 \%$ & $23 \%$ & $21 \%$ & $10 \%$ & $21 \%$ & $0 \%$ & $6 \%$ \\
\hline Imagen & $0 \%$ & $0 \%$ & $13 \%$ & $0 \%$ & $33 \%$ & $33 \%$ & $20 \%$ \\
\hline Miedo & $4 \%$ & $0 \%$ & $9 \%$ & $20 \%$ & $22 \%$ & $27 \%$ & $18 \%$ \\
\hline $\begin{array}{l}\text { Barreras del } \\
\text { momento }\end{array}$ & $0 \%$ & $0 \%$ & $20 \%$ & $18 \%$ & $15 \%$ & $13 \%$ & $35 \%$ \\
\hline $\begin{array}{l}\text { No percibe } \\
\text { beneficios }\end{array}$ & $4 \%$ & $0 \%$ & $2 \%$ & $2 \%$ & $22 \%$ & $48 \%$ & $22 \%$ \\
\hline
\end{tabular}

En la tabla 5 se muestra la descripción que los jóvenes hacen de los motivos para consumir alcohol. Encontramos que en el grado quinto la categoría más mencionada fue la de estrategias de afrontamiento, en los grados sexto y séptimo la de recursos personales, en el grado octavo la de presión externa, en el grado noveno la de motivos sociales y en el grado once la de beneficios percibidos.

En la categoría de presión externa se observa una disminución del porcentaje entre quinto y séptimo y el grado once tiene el menor porcentaje con un $8 \%$ de respuestas que hacen referencia a la influencia de los amigos como motivo para tomar. La categoría de imagen muestra una tendencia a incrementar con la edad, a excepción del grado octavo, en donde se presenta una disminución. Para quinto, solo un $3 \%$ de respuestas mencionaban el aparentar ante los demás como motivos de consumo, mientras que para once un $26 \%$ de respuestas estaban relacionadas con lo que pudieran pensar los demás, si aceptaba o no tomar alcohol.

En permisos de momento se observa que en séptimo esta categoría tiene gran peso para los estudiantes; ya que el $50 \%$ de las respuestas nombraban algunos de los motivos de esta categoría. 
En cuanto al consumo de alcohol como una estrategia de afrontamiento, los resultados muestran que los cursos que más hacen referencia a esa categoría son noveno, quinto y décimo. En los grados de sexto, séptimo y once ningún estudiante menciona que una persona de su edad pueda tomar para afrontar un problema o manejar sus emociones.

Los beneficios percibidos son más mencionados por los cursos de décimo y once y menos mencionados en el grado sexto (tabla 5). Por otra parte, la categoría de recursos personales tiende a aumentar entre los grados quinto a séptimo, llegando al porcentaje más alto en séptimo. Este disminuye entre los grados octavo y noveno, para volver a aumentar en décimo y once. Según los resultados, en el grado séptimo es donde más se percibe la ausencia de recursos personales como motivo de consumo. En el grado noveno ningún estudiante hizo referencia a esta categoría (tabla 5). En el grado séptimo se presenta el mayor porcentaje de estudiantes que señalan la pérdida de control frente a la bebida como una razón para que un joven de su edad consuma. El grado once presenta el menor porcentaje de estudiantes que señalan esta categoría como motivo de consumo. Se observa que en la categoría de experimentación, el porcentaje de respuestas tiende a disminuir a partir del grado noveno y tiene mayor peso en el grado sexto. Los estudiantes que menos mencionan la curiosidad y el deseo de vivir nuevas experiencias como razones para aceptar tomar alcohol son los de quinto y séptimo. No hay ningún curso que no haga referencia a esa categoría como motivo de consumo.

Por último, los grados con los estudiantes de menor edad, a excepción del grado séptimo, hacen menor referencia a la categoría de motivos sociales que los grados más altos. En general se percibe que con el tiempo esa categoría comienza a adquirir mayor importancia entre los participantes como razón para consumir alcohol.
Tabla 5.

Porcentajes de cada curso en las categorías de motivos para consumir.

\begin{tabular}{|c|c|c|c|c|c|c|c|}
\hline \multirow[t]{2}{*}{ Categoría } & \multicolumn{7}{|c|}{ Curso } \\
\hline & $5^{\text {to }}$ & $6^{\text {to }}$ & $7^{\mathrm{mo}}$ & $8^{\mathrm{vo}}$ & $9^{\text {no }}$ & $10^{\mathrm{mo}}$ & 11 \\
\hline $\begin{array}{l}\text { Presión } \\
\text { externa }\end{array}$ & $17 \%$ & $13 \%$ & $9 \%$ & $26 \%$ & $13 \%$ & $13 \%$ & $8 \%$ \\
\hline Imagen & $3 \%$ & $11 \%$ & $15 \%$ & $13 \%$ & $16 \%$ & $17 \%$ & $26 \%$ \\
\hline $\begin{array}{l}\text { Permisos del } \\
\text { momento }\end{array}$ & $5 \%$ & $0 \%$ & $50 \%$ & $14 \%$ & $14 \%$ & $14 \%$ & $5 \%$ \\
\hline $\begin{array}{l}\text { Estrategias } \\
\text { de afronta- } \\
\text { miento }\end{array}$ & $26 \%$ & $0 \%$ & $0 \%$ & $17 \%$ & $35 \%$ & $22 \%$ & $0 \%$ \\
\hline $\begin{array}{l}\text { Beneficios } \\
\text { percibidos }\end{array}$ & $5 \%$ & $3 \%$ & $14 \%$ & $11 \%$ & $7 \%$ & $18 \%$ & $42 \%$ \\
\hline $\begin{array}{l}\text { Recursos } \\
\text { personales }\end{array}$ & $12 \%$ & 185 & $35 \%$ & $6 \%$ & $0 \%$ & $12 \%$ & $18 \%$ \\
\hline $\begin{array}{l}\text { Pérdida de } \\
\text { control }\end{array}$ & $11 \%$ & $11 \%$ & $26 \%$ & $15 \%$ & $15 \%$ & $15 \%$ & $7 \%$ \\
\hline $\begin{array}{l}\text { Experimen- } \\
\text { tación }\end{array}$ & $9 \%$ & $24 \%$ & $9 \%$ & $16 \%$ & $16 \%$ & $14 \%$ & $12 \%$ \\
\hline $\begin{array}{l}\text { Motivos } \\
\text { sociales }\end{array}$ & $0 \%$ & $3 \%$ & $16 \%$ & $6 \%$ & $22 \%$ & $28 \%$ & $25 \%$ \\
\hline
\end{tabular}

\section{Discusión}

El objetivo de este estudio fue analizar las posibles motivaciones por las que los jóvenes consumen o no alcohol y cómo cambian estos motivos a medida que crecen. Además, encontrar puntos de encuentro en los diferentes cursos e identificar variables de intervención para la prevención del consumo de alcohol. A partir del análisis de las respuestas dadas por los jóvenes, se observó que en el proceso de toma de decisiones entran en juego representaciones sociales relacionadas con el imaginario de una persona que consume alcohol, el contexto en particular en el que se encuentren y las claves contextuales que le indiquen si el consumo es aprobado o desaprobado en ese momento en particular, tal como lo mencionan Gerrard, Gibbons, Brody, Murry \& Wills (2006) y Pascual, (2002). Así el análisis de una decisión de consumo ante un ofrecimiento depende de la situación en la que se encuentre el joven y no solo de un proceso de reflexión acerca de la peligrosidad de la sustancia. 
En el análisis de los motivos de consumo y no consumo se encuentra que las respuestas de los estudiantes reflejan la cultura de consumo de la comunidad a la que estos pertenecen y se nutren de lo que han visto y experimentado en su relación directa e indirecta con el alcohol (Donovan et ál., 2009; Martino et ál., 2006). Así, se encuentran particularidades en las respuestas de los estudiantes y, también, creencias generales y prototipos sociales del consumidor de alcohol que acompañan la motivación que el joven tenga para consumir o no consumir (Spijkerman, Van Den Eijnden, Overbeek, Engels, 2007; Andrews et ál., 2008). Encontramos que el alcohol es reconocido como un objeto de identificación e integración grupal, que sirve para estrechar y fortalecer las relaciones existentes (Kloep et ál., 2001; Moral y Ovejero, 2005).

Tanto en los motivos para consumir como en los motivos para no consumir se repiten categorías tales como las de imagen, recursos personales y pérdida de control, mostrando que dos jóvenes pueden utilizar un mismo motivo como razón para consumir o no consumir. Esto señala que las diferencias individuales representadas en variables tales como: la personalidad, la historia personal, el contexto y las estrategias de afrontamiento de cada persona influyen en las motivaciones de los jóvenes para tomar alcohol. Por otra parte, en la clasificación de los motivos de consumo o no consumo de alcohol se encontró que estos se pueden agrupar en aquellos que tienden a ser más duraderos en el tiempo e intrínsecos y en aquellos motivos que reflejan más una circunstancia en particular, una restricción o un facilitador externo que impide o permite que el joven consuma alcohol en el momento.

Dentro de los principales motivos para no consumir alcohol encontramos que los recursos personales, tales como pensar que no es adecuado hacerlo, tienen gran relevancia en esta muestra. Es decir, que la formulación moral de lo que implica consumir alcohol es una razón a la que le otorgan gran peso; tal vez debido a que son estudiantes de un colegio católico donde los principios morales son parte de la educación integral de los jóvenes. Se evidenció, asimismo, que la salud física y las consecuencias de consumir alcohol son elementos relevantes en el desarrollo de las motivaciones que los jóvenes aprecian en cuanto al consumo de alcohol. En el cual, piensan que al beber puede afectarse esa parte del ser humano, motivo suficiente para no consumir. Se valora la salud física y mental de la persona; además, se le teme a los daños que se puedan presentar, es decir, hay cierta percepción de riesgo que puede llegar a disminuir la intención de consumo.

Otras categorías reiteran que, lo encontrado por la literatura y las investigaciones en donde se señalan la percepción de riesgo, percepción de beneficios, el consumo de pares, la presión directa e indirecta, la experimentación y las influencias normativas, se convierten en variables que están directamente relacionadas con la decisión de consumo de los jóvenes (Bekman et ál., 2011; Comasco et ál., 2010; Moral y Ovejero, 2005; Trujillo et ál., 2007). Asimismo, se encuentra la variable de control como una de las más mencionadas por los adolescentes. Se resalta que la percepción que los jóvenes tienen sobre el grado de control frente a la bebida está asociada con la motivación de consumo. Los resultados evidencian la ambivalencia que los adolescentes viven en cuanto al uso de esta sustancia, el cual les genera curiosidad y deseo de saber qué es y qué ocurre, pero les da miedo enfrentarse a consecuencias que se les salgan de las manos.

En cuanto a la evolución que tienen estas categorías, encontramos que tal como lo sugiere el estudio de Cranford, et ál. (2010), a medida que los jóvenes comienzan a crecer tiende a disminuir el pensamiento de oposición y rechazo a las sustancias, se comienza a flexibilizar la postura de los adolescentes y se vuelve más relativa y sujeta a una circunstancia en particular. Esto se debe a la retroalimentación que van viviendo a partir del consumo experimental, el cual disminuye la percepción de riesgo frente a la sustancia. Por ello, a medida que crecen, padres y figuras significativas comienzan a aprobar más el consumo en los jóvenes o a una preparación mental que disminuye distorsiones cognitivas y les permite tomar una decisión de consumir sin sentir que se están traicionando.

Como hallazgos generales se observa que, en los motivos para no consumir, los recursos personales, las normas y las leyes y el daño a la salud física, 
tienden a mantenerse en todos los cursos con porcentajes altos de respuestas, mostrando que independientemente de la edad o el grado al que el joven permanezca, estas categorías representan razones que hacen que los jóvenes rechacen un ofrecimiento de consumo de alcohol. Las categorías de imagen, miedo, barreras del momento y no percibe beneficios, tienden a aparecer en séptimo y a presentarse más en los cursos mayores, con un porcentaje nulo o muy pequeño de estudiantes de cursos de quinto y sexto. Eso se puede dar porque a medida que crecen, los jóvenes van ampliando el marco de alternativas frente al consumo y tienden a flexibilizar más el pensamiento frente a la posibilidad de tomar alcohol.

Vale la pena resaltar que en los cursos menores, el miedo a las consecuencias de consumir alcohol no se evidencia tan claramente como en los cursos mayores. Esto puede deberse a que los mayores probablemente ya han experimentado con el alcohol y tal vez han tenido malas experiencias con este. Por otra parte, en muchas de las categorías se encuentra que séptimo y octavo eran cursos que representaban la excepción de la tendencia. Esto se relaciona con lo encontrado en las investigaciones que muestran que los grados séptimo y octavo representan puntos en los que los estudiantes comienzan a cambiar su postura frente al consumo en cuanto comienzan a tener mayor experiencia directa con el mismo y se ven en la necesidad de reevaluar sus creencias (Faden 2006; Oliva, Parra, y Sánchez, 2008).

Por otra parte, hay grupos que muestran mayor similitud en los porcentajes de las categorías, estos grupos son, por un lado, quinto y sexto y, por otro lado, décimo y once; lo cual puede indicar etapas similares de desarrollo y de socialización. Llama la atención que algunas categorías muestran porcentajes muy altos en algunos cursos y aparecen de manera reiterativa en las respuestas de los estudiantes, mientras que estas mismas categorías pueden no mencionarse en otros grados. Esto puede significar que los motivos y las creencias del consumo de alcohol pueden estar más asociados con procesos de socialización y dinámicas de interacción cultural, familiar y social, particular de cada curso, que a cambios relacionados con un proceso de maduración.

Finalmente, en términos de las implicaciones del estudio para los programas de prevención, el análisis de las categorías muestra la importancia de desestimular asociaciones culturales que generan una percepción de aprobación del consumo en menores de edad y que hacen que los jóvenes hagan excepciones de consumo de alcohol en reuniones sociales, familiares, celebraciones personales o cuando están en fiesta con amigos.

Se resalta la necesidad de que los programas preventivos inicien a una edad temprana, años antes de que los jóvenes comiencen a experimentar con el consumo y la necesidad de direccionarlos a las familias y comunidades para generar modelos adecuados de consumo de alcohol, además de un cambio de postura frente al uso de esta sustancia en menores de edad. Por otro lado, se debe buscar aumentar la responsabilidad que tienen las figuras significativas en una posterior decisión de consumo de alcohol del adolescente.

Las categorías construidas también señalan la pertinencia de realizar acciones preventivas que apunten a aumentar la motivación interna del joven para no consumir. Todo esto, a la luz de una postura personal de oposición al consumo, que sea coherente con sus valores y principios, como de un refuerzo a aquellos impedimentos circunstanciales, tales como: normatividad en la casa, dificultad de acceso, un castigo más fuerte para los consumidores menores de edad y mayor supervisión, que pueden hacer que el joven postergue su edad de inicio de consumo de alcohol y disminuya la frecuencia de consumo.

Para terminar, se establece la importancia de trabajar en las habilidades de afrontamiento y en los recursos personales, como factores protectores frente al consumo. Se sugiere reforzar la imagen personal como algo opuesto al consumo de alcohol, para desestimar las actitudes favorables que se tengan y promover la idea de que el consumo de esta sustancia puede disminuir el grado de control que tienen sobre su vida. Se reitera la importancia de generar programas de prevención donde se 
identifiquen los beneficios percibidos del alcohol y se busquen alternativas para conseguirlos de otra forma. Otro resultado importante para el ámbito de la prevención es la necesidad de buscar programas que tengan en cuenta variables como la personalidad, las historias indirectas de consumo, estrategias de afrontamiento y particularidades de los jóvenes, para que puedan responder a necesidades específicas de un grupo de personas con características similares. La mayoría de programas de prevención no tienen en cuenta diferencias personales que puedan hacer que un motivo de consumo en un adolescente se convertirá en un motivo de no consumo para otro adolescente. Finalmente, se hace evidente que los programas de prevención deben comenzar a contextualizar y especificar situaciones que facilitan el consumo de alcohol en los jóvenes, en las cuales, estos se dan permisos para tomar. Adicionalmente, intervenir en el desarrollo de habilidades, para situaciones concretas, tales como: fiestas, celebraciones y tiempo de ocio.

\section{Limitaciones del estudio}

Al ser un estudio descriptivo no permite establecer el grado de asociación ni la significancia de las relaciones encontradas. Asimismo, debido a que los jóvenes de la muestra presentan unas características sociodemográficas particulares, es necesario replicar la investigación en otro tipo de población para ver el grado de generalización de los resultados.

\section{Referencias}

Anderson, K., Grunwald, I., Bekman, N., Brown, S. \& Grant, A. (s.f). Motives and expectancies for use and nonuse in adolescence. Addictive Behaviors, 36(10), 972-979.

Anderson, K., Ramo, D., Cummins, K. \& Brown, S. (2010). Alcohol and drug involvement after adolescent treatment and functioning during emerging adulthood. Drug and alcohol dependence, 107, 171-181.

Andrews, J., Hampson, S., Barklet, M., Gerrard, M. \& Gibbons, F. (2008). The effect of early cognitions on cigarette and alcohol use during adolescence. Psychology of Addictive Behaviors, 22(1), 96-106.

Bekman, N. M., Anderson, K. G., Trim, R. S., Metrik, J., Diulio, A. R., Myers, M. G. \& Brown, S. A. (2011). Thinking and drinking: alcoholrelated cognitions across stages of adolescent alcohol involvement. Psychology of Addictive Behaviors. Doi: 10.1037/a0023302.

Comasco, E., Berglund, K., Oreland, L. \& Nilsson, K. W. (2010). Why do adolescents drink? Motivational patterns related to alcohol consumption and alcohol-related problems. Substance Use \& Misuse, 45(10), 1589-1604.

Cranford, J., Zucker, R., Jester, J., Puttler, L. \& Fitzgerald, H. (2010). Parental alcohol involvement and adolescent alcohol expectancies predict alcohol involvement in male adolescents. Psychology of addictive behaviors, 24(3), 386-396.

Donovan, E., Molina, B. \& Kelly, T. (2009). Alcohol outcome expectancies as socially shared and socialized beliefs. Psychology of Addictive Behaviors, 23, 248-259.

Epler, A., Sher, K. \& Piasecki, T. (2009). Reasons for abstaining or limiting drinking: a developmental perspective. Psychology of Addictive Behavior, 23, 428-442.

Faden, V. (2006). Trends in initiation of alcohol use in the United States. Alcoholism: Clinical and Experimental Research, 30(6), 1011-1022.

Gerrard, M., Gibbons, F. X., Lane, L. S., Wills, T. A., Brody, G. \& Conger, R. D. (2004). Context and cognitions: environmental risk, social influence and adolescent substance use. Personality and Social. Psychology Bulletin, 30, 1048-1061.

Gerrard, M., Gibbons, F. X., Brody, G. H., Murry, V. M. y Wills, T. A. (2006). A theory-based dual focus alcohol intervention for preadolescents: social cognitions in The Strong African 
American Families Program. Psychology of Addictive Behaviors, 20, 185-195.

Kloep, M., Hendry, L., Ingerbrigsten, J., Glendinning, A. \& Espness, G. (2001). Young people in drinking societies? Norwegian scottish and swedish adolescents' perception of alcohol use. Health Education Research, 16, 279, 291.

Lara, C. (2009). Diseño del cuestionario de creencias referidas al consumo de alcohol para jóvenes universitarios. Diversitas, 5(2), 337-347.

Litt, D. M. \& Stock, M. L. (2011). Adolescent alcohol-related risk cognitions: the roles of social norms and social networking sites. Psychology of Addictive Behaviors. Doi: 10.1037/a0024226.

Martino, S., Collins, R., Ellickson, P., Schell, T. \& McCaffrey, D. (2006). Socio-environmental influences on adolescents' alcohol outcome expectancies: a prospective analysis. Addiction, 101(7), 971-983.

Mason, W., Toumbourou, J. W., Herrenkohl, T. I., Hemphill, S. A., Catalano, R. F. \& Patton, G. C. (2011). Early age alcohol use and later alcohol problems in adolescents: Individual and peer mediators in a bi-national study. Psychology of Addictive Behaviors, 1939-1501.

Masten, A. S., Faden, V. B., Zucker, R. A. \& Spear, L. P. (2009). A developmental perspective on underage alcohol use. Alcohol Research \& Health, 32(1), 3-15.

Moral, M. y Ovejero, A. (2005). Análisis diferencial por niveles de edad de las actitudes hacia el consumo de sustancias psicoactivas en adolescentes españoles. Revista Interamericana de Psicología, 39(3), 325-338.

Moral, M., Rodríguez, F. y Sirvent, C. (2004). Motivadores de consumo de alcohol en adolescentes: análisis de diferencias intergénero y propuesta de un continuum etiológico Spiral. Adicciones, 17(2), 105-120.
Odgers, C., Caspi, A., Nagin, D., Piquero, A., Slutske, W., Milne, B., Dickson, N., Poulton, R. \& Moffit, E. (2008). Is it important to prevent early exposure to drugs and alcohol among adolescents? Psychologycal Science, 19(20), 1037-1043.

Oliva, A., Parra, A. y Sánchez, I. (2008). Consumo de sustancias durante la adolescencia: trayectorias evolutivas y consecuencias para el ajuste psicológico. International Journal of Clinical and Health Psychology, 8(1), 153-169.

Pascual, F. (2002). Percepción del alcohol entre los jóvenes. Adicciones, 14(1), 123-132.

Sanabria, A. (2010). Factores psicosociales de riesgo a conductas problemáticas en jóvenes infractores y no infractores. Diversitas, 6(2), 257-274.

Simons-Morton, B. (2007). Social influences on adolescent substance use. American Journal of Health Behavior, 31(6), 672-684.

Spikerman, R., Van Den Eijnden, R., Overbeek, G. \& Engels, R. (2007). The impact of peer and parental norms and behavior on adolescent drinking: the role of drinker prototypes. Psychology and Health, 22(1), 7-29.

Trujillo, A. (2009). Percepción de riesgo, estrés y consumo de drogas: estudio transcultural entre adolescentes de Barcelona y Bogotá (tesis doctoral).

Trujillo, A., Forns, M. y Pérez, A. (2007). Uso de sustancias y percepción de riesgo: estudio comparativo entre jóvenes de Bogotá y Barcelona. Adicciones, 19, 179-190.

Trujillo, A., Pérez, A. y Scopetta, O. (2011). Influencia de variables del entorno social sobre la ocurrencia de situaciones problemáticas asociadas al consumo de alcohol en adolescentes. Adicciones, 23, 349-356.

White, A. (2003). Substance use \& adolescent brain development. Youth Studies Australia, 22(1), 39. 